with a short list of selected references which will give ready access to the appropriate literature.

The work is conveniently divided into three sections. Under "Physiology of the Rumen" consideration is given to the movements and nervous control of the stomach, rumination, eructation, absorption and the flow of digesta. It is interesting to note that the phenomena associated with rumination, in spite of intensive, imaginative and ingenious research, still presents many unsolved problems. So too does the complex sequence involved in eructation-this latter function is especially important as its failure leads swiftly to the fatal consequence of hoven or bloat, a major disease in both dairy and beef cattle.

In the section "Metabolism in the Rumen" a brief outline of rumen microbiology is given, accounts of the fate of carbohydrates, proteins and lipids, and discussions of special techniques applicable to these fields. The microbiota of the rumen must surely be one of the most fascinating collections of organisms in a natural habitat; the delicately poised system in the rumen, so easy of access for research, is potentially a most fertile source of material for the ecologist who is interested in the dynamics of natural microbial populations.

The third section, on "Ruminant Nutrition", makes no attempt to cover the conventional fields' which are adequately treated in many text-books. Attention is focused on a few actively growing points, notably endocrine relations in metabolism and nutrition, and the synthesis of vitamins and microbial proteins in the rumen; also, the recent advances in ruminant physiology have awakened renewed interest in energy utilization, to which two chapters are devoted.

In spite of the limitations inseparable from any compilation based on a series of conference papers, this book will prove valuable to all students of ruminant nutrition and will provide a good background for readers interested in the metabolic diseases of ruminants.

I. W. McDonald

\section{TUMOUR METABOLISM}

The Glycolysis and Respiration of Tumours

By Alan C. Aisenberg. Pp. xiii + 224. (New York: Academic Press, Inc.; London : Academic Press, Inc. (London), Ltd., 1961.) 8 dollars.

HE present time seems quite appropriate for a dispassionate survey of our present-day knowledge and theorizing on the respiration and glycolysis of tumours. This subject begins with the original observations of Otto Warburg, made in 1923. The technical basis of these was important in itself: the introduction of the use of the thin slice of fresh tissue as experimental material and the development by Warburg of manometric methods based on those of Haldane and Barcroft; two of the most fruitful contributions to experimental biochemistry.

These are accepted. What is controversial concerns mainly the interpretation of these measurements in relation to the problem of cencer, and on this subject argument has tended to be hasty, intemperate and acrimonious, especially by the 'all-or-nothing' school, who will accept only a single key to cancer, and will discard everything less universal. On the other side of the picture, Warburg himself and his more fervent disciples have sometimes appeared to push their case too far against the oppos- ing weight of evidence, or at least to turn a blind eye to inconvenient experimental findings.

The primary beliefs of the Warburg school are briefly as follows: $(a)$ in relation to tumour growth, and to the neoplastic process in general, energyyielding metabolism is all-important; the rest is secondary. (b) While growing tissues, both embryonic and neoplastic, when maintained anaerobically have a high rate of glycolysis (that is, lactic acid formation from glucose), aerobically only the tumour tissues show strong glycolysis. (c) There is a disturbance in the respiration of tumours which is not only the cause of their aerobic glycolysis but also which is fundamental to the nature of cancer.

As Dr. Aisenberg clearly brings out in his very satisfactory review of the relevant factual information, it is quite easy to assemble data either in support of each of the above dogmas, or contrary to them. Indeed, of the 538 literature references cited, a fair proportion are regarded by their authors as providing decisive evidence one way or the other. The fact that honest and capable workers should find themselves so divided, even sometimes on the experimental findings themselves, is a good reason for the publication of this book, which presents an unbiased and reliable account of the work up to 1960 on the respiration and glycolysis of tumour tissue, and, where the comparison is available, on related normal tissues.

In spite of all the work summarized in this useful book, I am left with an opinion not essentially different from that which I put forward in The Cancer Review in 1931: "Perhaps it might even have been assumed to be improbable from the outset that such fine distinctions as exist between various types of malignant and non-malignant growths should be wholly explicable in terms of simple measurement of respiration and glycolysis". Surely the respiration of a tissue should not be assessed for this purpose solely in terms of its rate of oxygen uptake, when it is now very much clearer than when the above words were written that tissue respiration is merely the overall expression of an exceedingly complex chain of enzymic reactions, involving alternative substrates, alternative metabolic pathways, alternative co-enzymes, and alternative routes of energy utilization. Nor can the entity 'aerobic glycolysis' be conveniently divorced from the complex balance of enzymic activities and sub-cellular structure by means of which it is regulated. Finally, the control of synthetic reactions, especially of biosynthesis of protein and of nucleic acids, needs to be considered in relationship to what Krebs has called 'pacemaker' reactions, which, though all-important in regulation of cell metabolism, may constitute only a small part - though a determining one-in the overall crude picture of 'respiration and glycolysis'. The author of this carefully 'neutral' book might not perhaps be willing to go so far, but his well-balanced record of thirty-seven years of experiment, inference and controversy should assist towards a happy issue to this vexed question.

The book is generally excellently produced by the Academic Press, and is a publication of the Cancer Commission of Harvard University. Minor blemishes occur in the spelling of some names (Lipmann, p. 67; Stickland, p. 217); the date of Warburg's book, Stoffwechsel der Tumoren (p. 218), was 1926; "anaerobic glycolysis", attributed on p. 14, line 8, only to the very young embryo, should read "aerobic glycolysis". F. Dickens 